\title{
Quadruple Parabolic Aberration Curves Independently derived from Lanthanides in Samples of Leuco-granitic Gneiss and Seawater
}

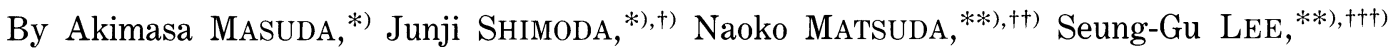 \\ and Mohammad B. SHABANI**),+†+) \\ (Communicated by Kazuo Yamasaki, M. J. A., Nov. 13, 1995)
}

\begin{abstract}
Four parabolic functions were derived separately for the first, the second, the third and the fourth tetrads of lanthanides in samples of leuco-granitic gneiss from Korea, and seawater from off the coasts of Shimane and Kochi prefectures, Japan. We conclude that relative lanthanide abundances in each of those samples are mathematically expressed by a set of four parabolic functions. The parabolic functions are applicable to the abundance ratio values as well as the values of aberration from the cusp-spanning lines. Both parabolic function systems are significant in different senses, but a set of four parabolas for "aberration" is convenient in visualizing the characteristics of lanthanide tetrad phenomenon observed for the sample. Difference in those characteristics can be interpreted to reflect uniquely the state of lanthanides present and/or the condition of genesis of the sample.
\end{abstract}

Key words : Lanthanides; tetrad effect; quadratic function; aberration; parabola; leuco-granitic gneiss; seawater; gneiss; water-rock interaction.

The lanthanide tetrad phenomena are discontinuities occurring at "one-quarter-filled", "half-filled" and "three-quarter-filled" subshell of 4 f electrons. ${ }^{1)-7)}$ For investigation of the lanthanide tetrad effect concerning the variation in concentration, high precision in lanthanide determination is required.

The highest reliability is guaranteed by isotope dilution mass spectrometry (IDMS) employing a thermal ionization mass spectrometer (TIMS) with an open-spacing ion source. This technique has a defect in that it is very difficult to determine the mono-isotopic lanthanides, $\mathrm{Pr}, \mathrm{Tb}, \mathrm{Ho}$ and Tm. Although ICP mass spectrometer can determine all lanthanides, the precision is not so high as IDMS by TIMS in general: this is particularly true for mono-isotopic lanthanides.

*) Department of Chemistry, University of ElectroCommunications, Chofu, Tokyo 182, Japan.

**) Department of Chemistry, The University of Tokyo, Hongo, Tokyo 113, Japan.

†) Present address: AT \& T Global Information Solution Japan, Ltd., Shibuya-ku, Tokyo 150, Japan.

${ }^{++}$Present address: Ministry of International Trade and Industry, Chiyoda-ku, Tokyo 100, Japan.

${ }^{++}$Present address: KIGAM, Taejeon, Korea.

${ }^{++\dagger)}$ Present address: Mitsubishi Materials Corp., Omiya 330, Japan.
According to our experience, the determination of Ho is most dependable among them. Some uncertainty can exist often for $\mathrm{Tm}$ and $\mathrm{Tb}$. Apart from analytical chemistry, a potential problem may be that $\mathrm{Tb}$ is the first element of the heavy lanthanides following the "middle" element, Gd. ${ }^{8)}$ It should be noted that intermingling of deteriorated data even for a single element would mislead consequences.

Masuda et al..$^{9)}$ have suggested that the lanthanide tetrad effect can be expressed mathematically by means of a quadratic function. In order to apply this method (TRIMY method) to the lanthanide abundance data obtained by ID-TIMS, it was necessary to assume a priori that the quadratic coefficients for the first and the second tetrads have the same value. Another essential point of this method is a hypothesis ${ }^{9)}$ that the quadratic functions for the first and the second tetrads intersect at the middle point between $\mathrm{Nd}$ and $\mathrm{Pm}$, while those for the third and the fourth tetrads at the middle point between Ho and Er. A major purpose of this study is to derive independently four parabolas without such assumptions, based on a series of good-quality lanthanide abundance determination.

After carefully checking the occasional possibility and extent of empirical errors, the following mathematical procedure has been judged to be reliable relative- 


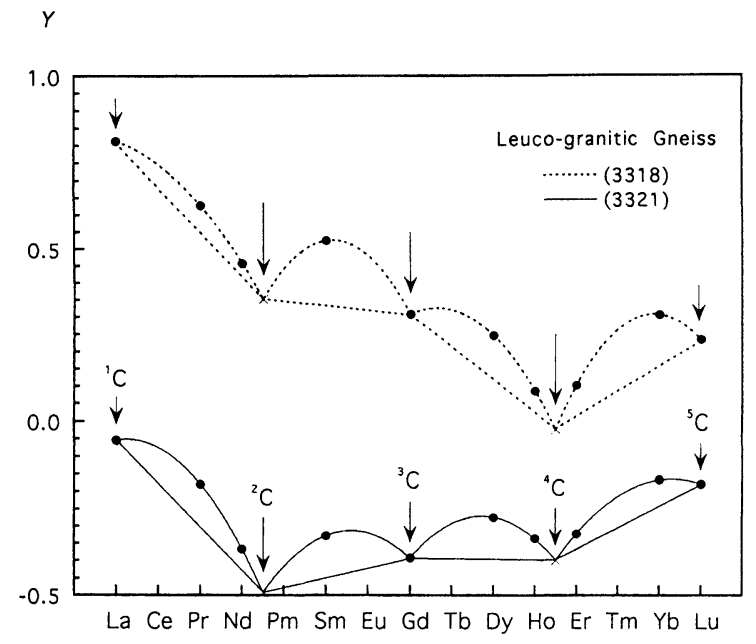

Fig. 1. Chondrite-normalized lanthanide patterns for two samples, 3318 and 3321, of leuco-granitic gneiss, Korea. ${ }^{13)}$ $Y=$ logarithm of abundance ratio of leuco-granitic gneiss to chondrite. ${ }^{14), 15)}$

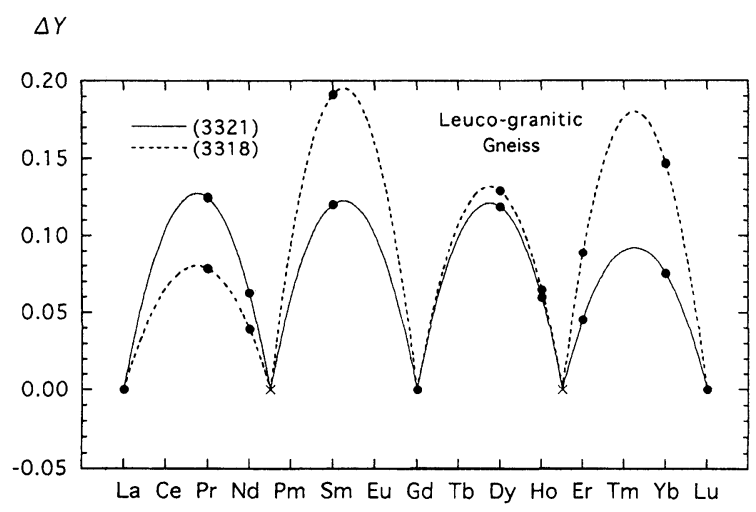

Fig. 2. The aberration diagram composed of four parabolas, for two samples of leucogranite (cf. Fig. 1).

ly free from the empirical errors: The parabolic function for the first tetrad is calculated from a combination of $\mathrm{La}, \mathrm{Pr}$ and $\mathrm{Nd}$. The third tetrad parabolic function is from Gd, Dy and Ho, while the fourth tetrad one from $\mathrm{Er}, \mathrm{Yb}$, and $\mathrm{Lu}$. Since Pm is not present in nature and Eu can be anomalous in valency, the second tetrad parabolic function involving Sm and $\mathrm{Gd}$ is calculated semi-independently after evaluation for the first tetrad subgroup. That is, it is assumed that the first tetrad function crosses the second tetrad one at the middle point between $\mathrm{Nd}$ and $\mathrm{Pm}$. (If $\mathrm{Eu}$ is regular in valency, this second one can be determined quite independently.)

Thus, one can obtain four independent parabolas from a set of lanthanide abundance data representing a certain individual specimen.
Passingly some words are added here about the "theoretical" aspect of lanthanide tetrad effect. Jørgensen $^{10)}$ and Kawabe ${ }^{11)}$ discussed this effect in terms of nephelauxetic phenomenon. Masuda ${ }^{12)}$ has proposed a simplified poly-axial steric symmetry model for the $4 \mathrm{f}$ electron clouds.

Rocks of leuco-granitic gneiss. Lee et al. ${ }^{13)}$ found an M-type tetrad phenomenon ${ }^{5)}$ in early Proterozoic leuco-granitic gneiss from Korea, in contrast with a W-type one ${ }^{5)}$ exemplified by seawater. Fig. 1 shows the lanthanide patterns for two whole-rock samples, 3318 and 3321, normalized by the Leedey chondrite. ${ }^{14), 15)}$ In their study, poly-isotopic lanthanides were first determined by ID-TIMS. Using those IDMS data as internal standards, the data acquired by ICP-MS for mono-isotopic lanthanides were used for determination of these lanthanides. Fig. 1 also depicts the cusp-spanning straight lines. The resulting aberration diagrams for two samples are shown in Fig. 2. It can be seen that the parabolas for the first, the second and the third tetrads for rock 3321 are quite similar to each other. The aberration maximum for the fourth tetrad is $75 \%$ of those for the other three tetrads. The high similarity in aberration curve means the high similarity in the quadratic coefficient, which is shown below. This means that the TRIMY assumption mentioned above is strictly valid in this case. In case of rock 3318 (broken-line curves), there is a considerable difference in the curve between the first and the second tetrads. It merits attention, however, that the second tetrad curve is similar to the fourth tetrad one. This may have something to do with the structure of electron clouds in the $4 \mathrm{f}$ orbital having the tetrahedral axial system which can reinforce the steric symmetry. ${ }^{12)}$ The difference in aberration characteristics between two rocks may reflect a difference in genetic process or reaction.

The third and fourth parabolas result in crossing at ${ }_{3} \mathrm{X}=3.495$ and 3.515 for rocks 3318 and 3321 , respectively. This confirms the validity of hypothesis about the crossing position in the TRIMY method. ${ }^{9)}$ The mathematical symbol ${ }_{3} \mathrm{X}$ here refers to the third sub-coordinate of four-segmented abscissa. ${ }^{20}$

Seawater samples. Lanthanides in seawater samples were determined cautiously by Shabani $e t a l .{ }^{16), 17)}$ by means of ICP-MS. They presented two sets of data, $\mathrm{S}_{1}$ and $\mathrm{S}_{2}$, on water (depth, $0-1 \mathrm{~m}$ from surface) collected from off the coast of Shimane prefecture. The concentrations for $\mathrm{S}_{2}$ (Fig. 3) are higher than those for $\mathrm{S}_{1}$ by factors of 1.3 1.6. Sample $\mathrm{S}_{1}$ appears to show 


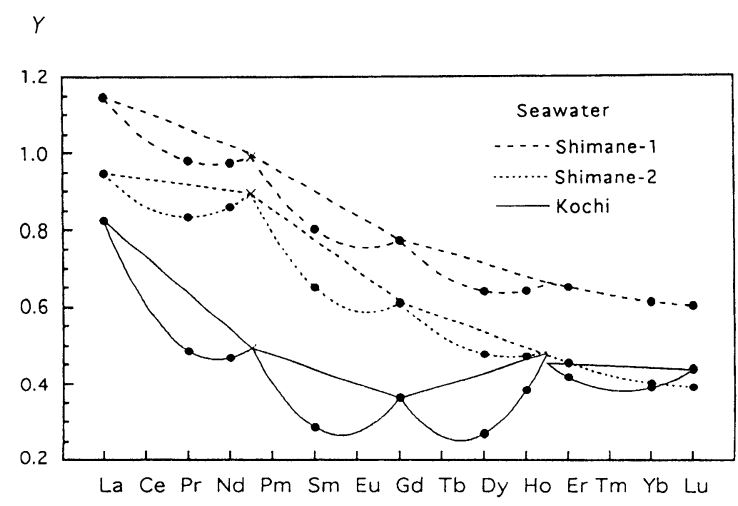

Fig. 3. Chondrite-normalized lanthanide patterns for samples of weawater. $Y=($ logarithm of abundance ratio of seawater to chondrite) plus 6 .

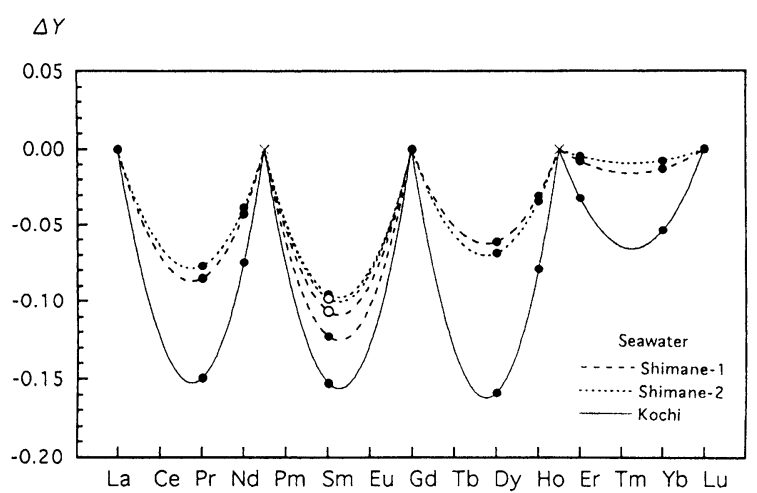

Fig. 4. The aberration diagram for seawater samples (cf. Fig. 3). ${ }^{16), 17)}$ Solid circles result from calculation based on ICP-MS, and open circles here from that based on ID-TIMS for Sm.

slightly larger aberrations (Fig. 4) than $\mathrm{S}_{2}$. This suggests that the concentration difference is mainly due to the relative difference in involvement of non-ionic fine particulate or dust and that there is a slight difference in chemical characteristics between the involved dusty material in two samples from the same location. The open circles for the second tetrad in Fig. 4 indicate the results of calculation based on determination by IDMS for Sm. Very small aberration for the fourth tetrad can be interpreted to indicate the relative immaturity of the dust material in the superfical water. Naturally these features reflect the state(s) of lanthanide present in water as well as the solid-liquid chemical reactions.

According to the mathematical calculation, the positions of crossing of the third parabola with the fourth one turn out to be 3.57 and 3.35 for two Shimane seawater samples, $S_{1}$ and $S_{2}$, respectively. This slight difference is ascribable to the small errors in chemical

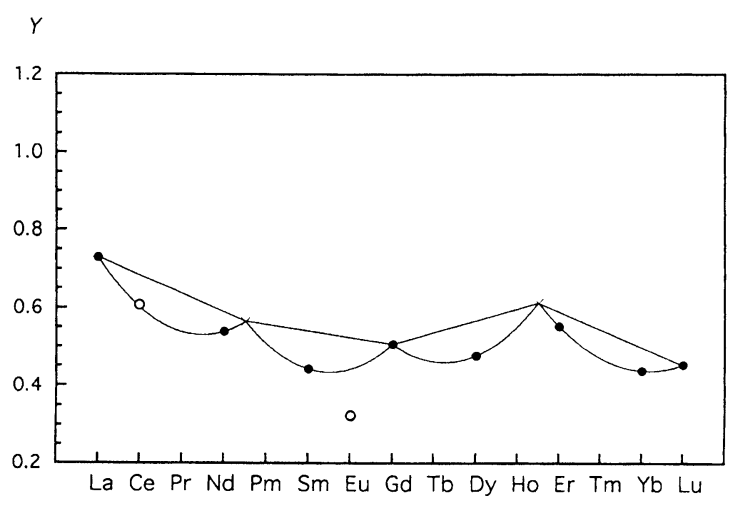

Fig. 5. Patterns of the ratio ${ }^{7), 19)}$ of lanthanide abundance in an aqueous phase to that in coexisting basaltic rock grains. $Y=(\operatorname{logarithm}$ of the foregoing ratio) plus 5 .

analysis. Note that the average is equal to 3.50 .

Shabani et al. ${ }^{17)}$ also determined carefully the lanthanides in seawater sampled from off the coast of Kochi prefecture (depth $320 \mathrm{~m}$ ) by ICP-MS. The chemical and mathematical analyses result in Figs. 3 and 4. The aberration parabolas (Fig. 4) are substantially the same for the first, the second and the third tetrads. Also it can be seen that the aberrations for Kochi seawater are larger than those for Shimane seawater. This difference is related obviously to the difference in depth of seawater sampled and indicates the difference in maturity of seawater and lanthanides therein.

The third parabola mathematically intersects the fourth one at ${ }_{3} \mathrm{X}=3.41$ in reasonable agreement ${ }^{20)}$ with other cases studied above.

Product of water-rock reaction. The lanthanide tetrad effect was found in the laboratory experiment on water-rock reaction. ${ }^{7), 19)}$ A typical case where lanthanides were determined with the high precision and safely free from incorporation of fine particles into the aqueous phase is exhibited in Fig. 5. In this experiment, a remarkable enhancement effect ${ }^{7), 19)}$ by $\mathrm{CO}_{2}$ gas or carbonate on lanthanide mobilization into the aqueous phase was observed. Since we have the data on multi-isotopic lanthanides only, the curves were mathematically obtained based on the TRIMY method. The corresponding aberration diagram (solidline parabolas) is presented in Fig. 6. Again the parabolic aberration curves are almost identical to each other for the first, the second and the third tetrads. The aberration maximum for the fourth tetrad is $80 \%$ of those for the three other ones. It is unknown whether the similarity of this numerical value to that observed for the fourth tetrad of leuco-granitic gneiss 


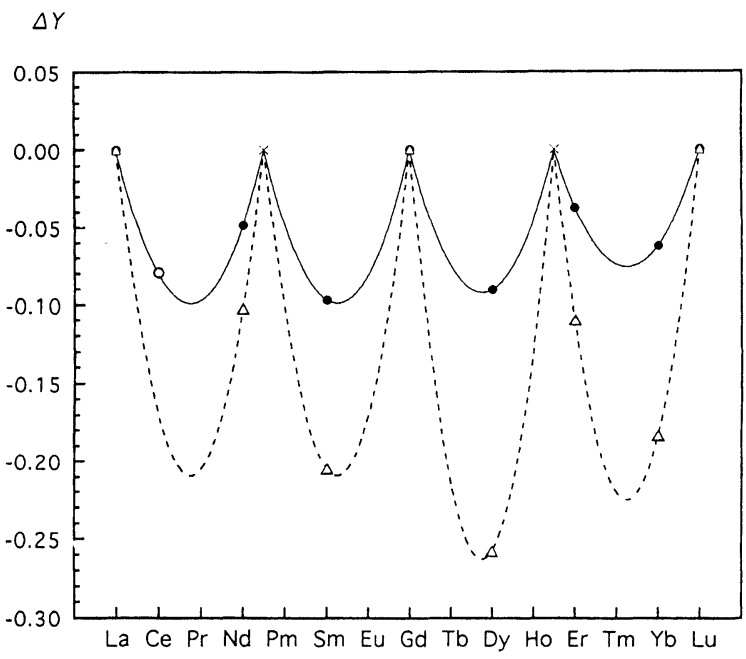

Fig. 6. The aberration diagram for products of water-rock interaction (cf. Fig. 5 and ref. 20).

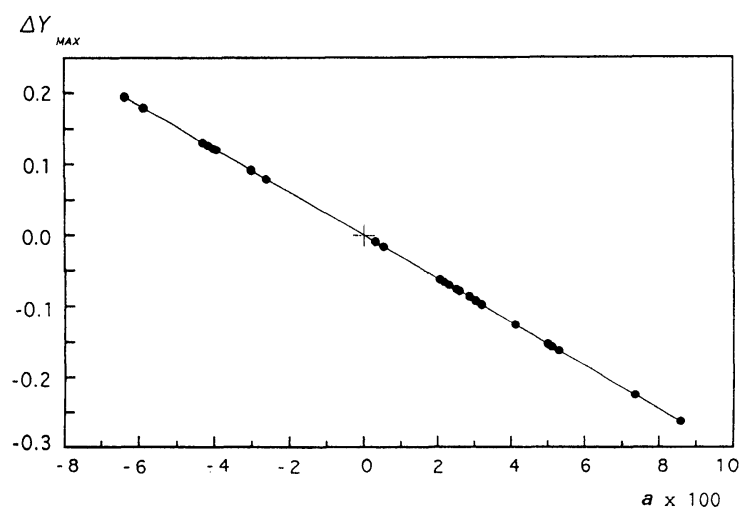

Fig. 7. Observed range of variation of the aberration maximum $\Delta Y_{\mathrm{MAX}}$ and quadratic coefficient $a$.

3321 has a physical meaning or not.

In Fig. 5, the open circles represent the observed ratios of $\mathrm{Ce}$ and $\mathrm{Eu}$. We observe that the circle for $\mathrm{Ce}$ falls on the curve mathematically drawn by the TRIMY method. This suggests that Ce is present as a trivalent ion in both the basaltic rock used and the aqueous phase under the condition of this experiment (two-week soakage with bubbling $\mathrm{CO}_{2}$ gas into the water). The bubbling of $\mathrm{CO}_{2}$ does not only enhance the $\mathrm{CO}_{2}$ pressure in the water but purges dissolved oxygen from the aqueous phase, preventing the oxidation of $\mathrm{Ce}(\mathrm{III})$ to tetravalent. Fitting the position of Ce to the "theoretical" curve can be taken to verify the validity of the TRIMY method. The negative deviation of the open circle for $\mathrm{Eu}$ can be explained in terms of partial presence of divalent $\mathrm{Eu}$ in feldspar ${ }^{18)}$ and differentiated feldspar dissolution on time. ${ }^{19)}$
In Fig. 6 is superimposed the aberration diagram derived by the TRIMY method for a ground water (broken-line curve) from Shin-Kobe tunnel. ${ }^{20)}$ In this case, all four parabolas appear to be almost similar to each other. In terms of absolute values, aberration is largest for this ground water running down the granitic wall rock.

It is worthwhile to note that the cusp-spanning patterns (Fig. 5 and Ref. 20) affected specifically by the positions of cusp points ${ }^{2} \mathrm{C}$ and ${ }^{4} \mathrm{C}$ deviate notably from two trans-octad lines. This lack of parallelism between the cusp-spanning lines and the trans-octad lines can be an aspect connately related with the lanthanide tetrad effect.

As explained above, the quadratic function $f(\mathrm{x})=a$ $x^{2}+b x+c$ has been applied to express mathematically the lanthanide tetrad phenomenon. If $b / a$ is nearly constant, there should be a linear relation between quadratic coefficient $a$ and the aberration maximum, as substantiated in Fig. 7.

In summary, the intersection between the third and the fourth tetrad functions occurs at ${ }_{3} \mathrm{X}=3.5$, i.e., at the middle point between Ho and Er. Likewise the first tetrad function is inferred to intersect the second one at the middle point between $\mathrm{Nd}$ and $\mathrm{Pm}$. A fact observed for $\mathrm{Ce}$ in the laboratory experiment where $\mathrm{O}_{2}$ has been purged from the water-rock interaction system is a convincing evidence for this inference. The "middle-point" intersection under consideration implies that, in general, effects of vertically crossing equi-tri-axial system (octahedral) and "tetrahedrally" crossing equi-quadri-axial system (hexahedral) ${ }^{12)}$ are substantially the same in virtual efficiency to enhance the sphericity of $\mathrm{Ln}^{3+}$ ion.

A hypothesis concerning the quadratic coefficients of the first and the second parabolic functions is strictly valid sometimes but only roughly in other cases, indicating the different aspects (both simple and complex) of the phenomenon under consideration. It is ideal that the first parabolic function is independently derived. For this purpose, it is desirable that $\mathrm{Pr}$ is determined with precision comparable with those for poly-isotopic lanthanides determinable with ID-TIMS. If quadratic coefficients, $a_{1}$ and $a_{2}$, for the first and the second tetrads are considerably different from each other, the quadratic coefficient $a_{l}$ obtained by the TRIMY method ${ }^{9}$ turns out to be larger than the actual individual coefficients, $a_{1}$ and $a_{2}$. The degree of this bias is larger for $a_{1}$ than for $a_{2}$ (cf. ref. 20). The TRIMY method, however, would be sufficiently useful 
in practice. The adequacy of the resultant value for $a_{l}$ may be judged in comparison with $a_{3}$ and/or $a_{4}$,

It might be worthwhile to note that, so far as the cases studied here are concerned, the maximum aberration for the second tetrad is almost identical with either the third or the fourth one or both of them.

The lanthanide tetrad effect is invariably affected by the chemical condition surrounding the lanthanide ion such as presence of water (and carbon dioxide). At the same time, the following two factors are important in connection with the $4 \mathrm{f}$ electron clouds ${ }^{12}$; whether the lanthanide tetrad concerned belongs to the former octad $(Z \leqq 64)$ or the latter octad $(Z \geqq 64)$ and whether it is an odd-numbered tetrad or an even-numbered tetrad. ${ }^{2,, 8), 12)}$

Acknowledgements. Continued support by Prof. K. Yamasaki, M. J. A., for this series of studies is gratefully acknowledged. Our thanks are extended to Dr. M. Minami for her kind help.

\section{References}

1) Fidelis, I. K., and Siekierski, S. (1966) J. Inorg. Nucl. Chem. 28, 185-188.

2) Fidelis, I. K., and Mioduski, T. J. (1981) Structure and Bonding 47, Springer-Verlag, Berlin.

3) Peppard, D. F., Mason, G. W., and Lewey, S. (1969) J. Inorg. Nucl. Chem. 31, 2271-2272.
4) Masuda, A., and Ikeuchi, H. (1979) Geochem. J. 13, 271-277.

5) Masuda, A., Kawakami, O., Dohmoto, Y., and Takenaka, T. (1987) Geochem. J. 21, 119-124.

6) Kagi, H., Dohmoto, Y., Takano, S., and Masuda, A. (1993) Chem. Geol. 107, 71-82.

7) Minami, M., Matsuda, N., and Masuda, A. (1995) Proc. Japan Acad. 70B, 10-14.

8) Masuda, A. (1995) Proc. Japan Acad. 71B, 67-71.

9) Masuda, A., Matsuda, N., Minami, M., and Yamamoto, H. (1994) Proc. Japan Acad. 70B, 169-174.

10) Jørgensen, C. K. (1970) J. Inorg. Nucl. Chem. 32, 31273128.

11) Kawabe, I. (1992) Geochem. J. 26, 309-335.

12) Masuda, A. (1995) Proc. Japan Acad. 71B, 165-169.

13) Lee, S-G., Masuda, A., and Kim, H-S. (1994) Chem. Geol. 114, 59-67.

14) Masuda, A., Nakamura, N., and Tanaka, T. (1973) Geochim. Cosmochim. Acta 37, 239-248.

15) Masuda, A. (1975) Geochem. J. 9, 183-184.

16) Shabani, M. B., Akagi, T., Shimizu, H., and Masuda, A. (1990) Anal. Chem. 62, 2709-2714.

17) Shabani, M. B., Akagi, T., and Masuda, A. (1992) Anal. Chem. 64, 737-743.

18) McKay, G. (1982) Lunar Planet. Sci. 8, 493-494.

19) Matsuda, N. (1992) Master Thesis, The University of Tokyo.

20) Masuda, A., Shimoda, J., and Yamamoto, H. (1995) Proc. Japan Acad. 71B, 208-213. 